\title{
Evolução dos conhecimentos sobre doenças imunopreveníveis de alunos no curso de Medicina da Universidade Estadual de Londrina
}

\section{Improvement of the knowledge about immuno-preventable diseases of students at Medical School of Universidade Estadual de Londrina}

\author{
Márcia Cristina Bento Moreira ${ }^{1}$; Gerson Zanetta de Lima²
}

\begin{abstract}
Resumo
Profissionais de saúde constituem um grupo de especial preocupação em relação às doenças infecciosas, por serem mais expostos à infecção e representarem risco para si e para seus pacientes, por isso um esquema especial de vacinação tem sido recomendado para esta população nos últimos anos. O presente estudo teve como objetivos a avaliação da situação vacinal e a evolução dos conhecimentos acerca de doenças imunopreveníveis por alunos matriculados no Curso de Medicina da Universidade Estadual de Londrina (UEL) em 2006. Foram utilizados um questionário auto-aplicável e uma entrevista com os alunos da $1^{\mathrm{a}}, 3^{\mathrm{a}}$ e $6^{\mathrm{a}}$ séries, com a finalidade de verificar as vacinas por eles recebidas e os conhecimentos acerca das doenças infectocontagiosas a que estão expostos. Foram incluídos $219(91,2 \%)$ alunos dos 240 matriculados, sendo $75(34,2 \%), 78(35,6 \%)$ e $66(30,1 \%)$ da $1^{\mathrm{a}}, 3^{\mathrm{a}}$ e $6^{\mathrm{a}}$ séries, respectivamente. Todos são brasileiros e $189(86,3 \%)$ relataram ter recebido, com certeza, as vacinas da infância; $134(61,2 \%)$ informaram apresentar cicatriz de BCG, $172(78,5 \%)$ referiram ter recebido vacinas nos últimos 5 anos. Formularam-se perguntas acerca de uma lista de doenças infecciosas e os resultados das respostas das três séries foram comparados e apresentaram diferenças significativas entre os alunos do início e os do fim do curso com relação ao número de respostas certas à $1^{\mathrm{a}}$ e à $2^{\mathrm{a}}$ questões $(\mathrm{p}<0,001)$; além disso $\mathrm{o}$ número de acertos foi crescente conforme a série. Os resultados demonstraram uma evolução positiva da cobertura vacinal com a progressão das séries, mas os valores de cobertura variaram conforme a vacina e, ademais, muitos alunos da $6^{a}$ série ainda apresentavam vacinação incompleta em relação à recomendada pelas autoridades sanitárias do país.
\end{abstract}

Palavras-chave: Universitários. Doenças imunopreveníveis. Imunizações. Cobertura vacinal.

\begin{abstract}
Health care professionals are part of a special group concerning infectious diseases, because they are more exposed to infections and represent enhanced risk to themselves and to their patients. Special vaccination schemes have been recommended for this group over the last years. This study was carried out to evaluate vaccine status and knowledge about immuno-preventable diseases among medical students of the State University of Londrina, Brazil, through a questionnaire and an interview about vaccines, answered by $1^{\text {st }}, 3^{\text {rd }}$ and $6^{\text {th }}$ grade medical students. Two hundred and nineteen $(91.2 \%)$ Brazilian students have been included from a total of 240 students officially registered: 75 (34.2\%), 78 (35.6\%) and $66(30.1 \%)$ were from the $1^{\text {st }}, 3^{\text {rd }}$, and $6^{\text {th }}$ grades, respectively. Most of them or $189(86.3 \%)$ reported to have received the vaccines from infancy; 134 (61.2\%) informed to have BCG scars; 172 (78.5\%) informed to have received vaccines in the last 5 years. The results were compared regarding the answers to four
\end{abstract}

\footnotetext{
1 Estudante do $5^{\circ}$ ano do curso de Medicina da Universidade Estadual de Londrina, bolsista IC-UEL. E-mail: marciacristinamoreira@yahoo.com.br.

2 Professor adjunto do curso de Medicina da Universidade Estadual de Londrina, orientador.
} 
questions about a list of infectious diseases. Significant differences were observed between students from the first year and those from the last year of the course, concerning right answers to the first and the second questions ( $\mathrm{p}<0.001$ ), with increased number of correct answers observed among students from the last year of the course. The results showed a positive evolution of the vaccine status, the values varying according to vaccine types, and many students of the $6^{\text {th }}$ grade continued to have incomplete vaccination as compared to the recommendations of Brazilian health authorities.

Key words: Medical students. Immuno-preventable. Diseases. Immunization. Vaccine coverage.

\section{Introdução}

A área de doenças infecciosas tem-se expandido continuamente, com o aparecimento de novas doenças e o reaparecimento de outras. Esforços no sentido de controlar as existentes, as emergentes e as re-emergentes têm sido crescentes nos últimos anos, e têm o objetivo de diminuir seu impacto na morbimortalidade. Os profissionais de saúde constituem um grupo de especial preocupação, uma vez que estão mais expostos ao contágio e, em consequiência, à doença. Propiciam, também, risco para seus pacientes, posto que podem ser a fonte destas doenças contagiosas, contribuindo para piorar sua condição clínica e sua qualidade de vida. Várias vacinas são hoje propostas para os profissionais de saúde (ADVISORY COMMITTEE ON IMMUNIZATION PRACTICES, 1997; ADVISORY COMMITTEE ON IMMUNIZATION PRACTICES, 2002; MEDEIROS; MARINO, 2000). Algumas são recomendadas e oferecidas gratuitamente pelo governo brasileiro e outras podem ser obtidas em clínicas especializadas que as distribuem comercialmente (BRASIL, 2002). A tendência é que o Programa Nacional de Imunizações (PNI) adote cada vez mais vacinas para esse grupo. A responsabilidade dos hospitais e das instituições de ensino para com esses profissionais fica cada vez mais caracterizada, uma vez que eles se expõem a doenças imunopreveníveis no ato da prestação de serviços a populações atendidas por essas instituições. Por outro lado, a cobertura vacinal de adultos alcançada no Brasil pelos programas já existentes e com vacinação gratuita está longe de atingir a meta de vacinação de $100 \%$ dos susceptíveis. Isso ocorre, em parte, por que a população desconhece as vacinas disponíveis, os riscos a que está exposta e muitas vezes as próprias doenças contra as quais estas vacinas são utilizadas (MALUF; ALDENUCCI, 2000). Pouco se têm investigado o conhecimento que alunos das áreas da saúde detêm em relação a estas questões, e há poucas publicações na literatura internacional e nacional (RIBEIRO, 2002). Este estudo constitui parte de uma investigação mais ampla e de mesma natureza realizada com alunos da área da saúde ingressantes na Universidade Estadual de Londrina (UEL) no ano de 2004 e teve por objetivo investigar a cobertura vacinal relatada e o conhecimento sobre vacinas e doenças imunopreveníveis entre os alunos da $1^{\mathrm{a}}, 3^{\mathrm{a}}$ e $6^{\mathrm{a}}$ séries do ano de 2006 do curso de Medicina da UEL.

\section{Casuística, Material e Métodos}

Foram sujeitos de investigação todos os alunos matriculados na $1^{\mathrm{a}}, 3^{\mathrm{a}}$ e $6^{\mathrm{a}}$ séries do curso de Medicina da UEL no ano de 2006, conforme dados oficiais da instituição.

Os alunos responderam a um questionário autoaplicável acerca de doenças infecciosas, vacinas e dados pessoais (idade, sexo, naturalidade, irmandade, cicatrizes de varicela ou de vacina BCG e informações das vacinas que receberam nos últimos 5 anos). Foram entrevistados a respeito de sua situação vacinal para Hepatite B, Sarampo, Caxumba, Rubéola, Difteria, Tétano e Varicela. Cada aluno foi convidado até duas vezes a participar e efetuou-se a busca ativa dos que não responderam. Esse procedimento teve por objetivo abranger todos os alunos matriculados nas séries mencionadas e só não foram incluídos apenas os que se negaram a participar. Todos os que participaram responderam afirmativamente à uma declaração de consentimento esclarecido, concordando em participar da pesquisa. 
Os resultados foram tabulados e analisados pelo programa Epi Info ${ }^{\mathrm{TM}}$ (CENTERS FOR DISEASE CONTROL AND PREVENTION, 2004). Utilizouse a contagem de frequiências para as respostas simples e a estatística de Kruskal-Wallis para a distribuição de freqüências de respostas corretas a quatro questões, propostas a partir de uma lista de doenças apresentada aos alunos.

\section{Resultados}

Foram avaliados $219(91,3 \%)$ alunos do total de 240 matriculados nas 3 séries, sendo $75(34,2 \%)$ do $1^{\circ}, 78(35,6 \%)$ do $2^{\circ}$ e $66(30,1 \%)$ do $6^{\circ}$ anos, respectivamente; $114(52,1 \%)$ eram mulheres. Eram naturais de Londrina 19 (8,7\%) e de outras cidades doEstado do Paraná 36 (16,4\%); todos eram brasileiros. Nenhum morou em zona rural até os dois anos de idade; $137(62.5 \%)$ procediam de cidades com mais de 100.000 habitantes e apenas $20(9,3 \%)$ procediam de cidades com menos de 10.000 habitantes.

Quanto ao número de irmãos, 177 (81\%) relataram ter um ou dois irmãos e, quanto à irmandade, eram o primeiro ou segundo filhos 191 (87\%). Quatro $(1,8 \%)$ alunos prestaram o serviço militar e nenhuma aluna relatou gravidez anterior ou atual; 35 (16\%) viajaram ao exterior nos últimos 5 anos, um $(0,4 \%)$ à Amazônia. Estas situações são associadas a orientação para recebimento de vacinas, mas os números encontrados não permitiram uma comparação de cobertura entre esses grupos.

Acerca da situação vacinal, $170(77,6 \%)$ alegaram certeza de possuir carteira de imunização, $32(14,6 \%)$ ignoravam. Referiram vacinas nos últimos 5 anos 172 $(78,5 \%)$ alunos; as mais comuns informadas foram as vacinas contra a difteria e o tétano tipo adulto ou dupla tipo adulto - dT $(\mathrm{n}=100,45,6 \%)$, contra a hepatite B $(n=144,65,7 \%)$ e contra a febre amarela $(n=59,26,9 \%)$. Informaram ter cicatrizes de alguma vacina que tivessem recebido ou de doença que tivessem tido 188 (87\%); destas 134 (61,1\%) eram cicatrizes de BCG. O comparativo das últimas vacinas recebidas relatadas foi estabelecido segundo o tipo e o total de alunos matriculados por série: dupla tipo adulto (dT) - 27 (35\%), 36 (46,1\%) e 37 (56\%); contra a febre amarela - 15 (20\%), $27(34,6 \%)$ e 17 (25,7\%); contra a hepatite B - $29(38,7 \%), 58(74,5 \%)$ e $56(84,8 \%)$; contra a raiva $2(2,3 \%), 7(8,9 \%)$ e 8 $(12,1 \%)$; contra o sarampo, a caxumba e a rubéola 0 $(0 \%), 1(1,2 \%)$ e $0(0 \%)$; outras vacinas $9(12 \%), 12$ $(15,4 \%)$ e $12(18,2 \%)$.

Questionados acerca de que recursos procurariam para serem vacinados, $162(74 \%)$ responderam corretamente (postos de saúde, clínicas particulares e sala de vacinas do Hospital de Clínicas de Londrina). Houve uma evolução deste conhecimento conforme a série. As respostas dos alunos da $1^{\mathrm{a}}, 3^{\mathrm{a}}$ e $6^{\mathrm{a}}$ séries, respectivamente, foram informadas corretamente por 48 (64\%), 61 (78,2\%) e $53(80,3 \%)$ alunos; parcialmente corretas por 6 (8\%), $5(6,4 \%)$ e $2(3 \%)$; incorretamente por $2(2,7 \%)$, $0(0 \%)$ e $2(3 \%)$ e não responderam $10(25,3 \%), 12$ $(15,4 \%)$ e $9(13,6 \%)$.

Solicitados a citar seis doenças às quais estariam expostos pela profissão, as mais frequentemente lembradas foram: hepatites, vírus da imunodeficiência humana (HIV)/aids, tuberculose e gripe (Tabela 1).

Tabela 1. Doenças infecto-contagiosas às quais estariam mais expostos pela profissão, mais lembradas por alunos matriculados na $1^{\mathrm{a}}, 3^{\mathrm{a}}$ e $6^{\mathrm{a}}$ séries do curso de Medicina da UEL no ano de 2006.

\begin{tabular}{l|l|l|l|l|l}
\hline Doença & $\mathbf{N}^{\mathbf{0}}$. citações & Doença & $\mathbf{N}^{\mathbf{0}}$. citações & Doença & $\mathbf{N}^{\mathbf{0}}$. citações \\
\hline Hepatites & $210(95,9 \%)$ & Meningites & $>20 \mathrm{e}<60(>9,1 \mathrm{e}<27 \%)$ & Outras & $<10(<4,5 \%)$ \\
\hline HIV/aids & $143(65,3 \%)$ & Pneumonias & $>20 \mathrm{e}<60(>9,1 \mathrm{e}<27 \%)$ & & \\
\hline Tuberculose & $137(62,5 \%)$ & Sarampo & $>20 \mathrm{e}<60(>9,1 \mathrm{e}<27 \%)$ & \\
\cline { 1 - 5 } Gripe & $87(39,7 \%)$ & Rubéola & $>20 \mathrm{e}<60(>9,1 \mathrm{e}<27 \%)$ & \\
\hline
\end{tabular}

Obs.: Algumas doenças foram citadas incorretamente, por pequeno número de alunos 
Frente a uma lista de 45 doenças infecciosas, apresentadas no Quadro 1, foram formuladas quatro perguntas:

1) Para quais destas doenças existem vacinas?

2) Para quais destas doenças existem soros ou imunoglobulinas?

3) Quais destas doenças já foram eliminadas ou controladas no Brasil pelo uso de vacinas?

4) Para quais destas doenças você foi vacinado?
As respostas corretas dos alunos das três séries às três primeiras questões foram comparadas pelo teste de Kruskal-Wallis, obtendo-se diferenças significativas para as respostas certas à $1^{\mathrm{a}} \mathrm{e}$ à $2^{\mathrm{a}}$ questões ( $p<0,001)$ e o número de acertos aumentou, conforme a série (Tabela 2 e Gráfico 1). Poucas foram as respostas incorretas nas três séries e não foi observada diferença significativa quando as respostas certas foram comparadas com as diferenças entre respostas certas e erradas para as três séries.
1. Aids (SIDA)
2. Angina (amidalite)
3. Câncer
4. Cólera
5. Diabetes
6. Difteria
7. Dengue
8. Diarréia
9. Doença de Chagas
10. Esquistossomose
11. Febre amarela
12. Febre tifóide
13. Gonorréia
14. Gripe
15. Hepatite A

16. Hepatite B

17. Hepatite C

18. Hepatite D

19. Hepatite E

20. Herpes

21. Herpes zoster

22. Hemófilo B (doença por)

23. Herpangina

24. D. Hansen (Lepra)

25. Lues (Sífilis)

26. Leishmaniose

27. Malária

28. Meningococo A (meningite)

29. Meningococo B (meningite)

30. Meningococo $\mathrm{C}$ (meningite)
31. Parotidite (caxumba)
32. Peste bubônica
33. Pneumococo (doença por)
34. Pneumonia
35. Poliomielite
36. Raiva
37. Resfriado
38. Rubéola
39. Sarampo
40. Tétano
41. Coqueluche (pertussis)
42. Toxoplasmose
43. Tuberculose
44. Varicela (Catapora)
45. Varíola

Quadro 1. Lista de 45 doenças infecciosas a respeito das quais foram formuladas quatro perguntas sobre imunoprevenção, para alunos matriculados em 2006 na $1^{\mathrm{a}}, 3^{\mathrm{a}}$ e $6^{\mathrm{a}}$ séries do curso de Medicina da Universidade Estadual de Londrina.

Tabela 2. Respostas corretas dadas por alunos matriculados em 2006 na $1^{\mathrm{a}}, 3^{\mathrm{a}} \mathrm{e} 6^{\mathrm{a}}$ séries do curso de Medicina da Universidade Estadual de Londrina às seguintes perguntas, feitas a partir da lista de doenças do Quadro 1: 1) Para quais destas doenças existem vacinas? 2) Para quais destas doenças existem soros ou imunoglobulinas? 3) Quais destas vacinas já foram eliminadas ou controladas no Brasil pelo uso de vacinas? 4) Para quais destas doenças você foi vacinado?

\begin{tabular}{|c|c|c|c|c|c|c|c|c|c|c|}
\hline Questão & & & 1 & & & & & 2 & & \\
\hline Resposta & Mín. & $\% 25$ & Méd. & $\% 75$ & Máx. & Mín. & $\% 25$ & Méd. & $\% 75$ & Máx. \\
\hline \multicolumn{11}{|l|}{ Série } \\
\hline 1 & 0 & 7 & 9 & 11 & 16 & 0 & 0 & 0 & 1 & 4 \\
\hline 2 & 0 & 9 & 11 & 13 & 17 & 0 & 0 & 1 & 2 & 4 \\
\hline 3 & 0 & 12 & 15 & 16 & 21 & 0 & 2 & 2 & 3 & 7 \\
\hline & & & & & & & & & & \\
\hline Questão & & & 3 & & & & & 4 & & \\
\hline Resposta & Mín. & $\% 25$ & Méd. & $\% 75$ & Máx. & Mín. & $\% 25$ & Méd. & $\% 75$ & Máx. \\
\hline \multicolumn{11}{|l|}{ Série } \\
\hline 1 & 0 & 1 & 2 & 2 & 3 & 0 & 3 & 5 & 7 & 13 \\
\hline 2 & 0 & 1 & 2 & 2 & 3 & 0 & 5 & 6,5 & 8 & 13 \\
\hline 3 & 0 & 1 & 2 & 3 & 3 & 0 & 7 & 8,5 & 10 & 13 \\
\hline
\end{tabular}

Legenda: Mín.= valores mínimos; \%25 = 1 quartil; Méd.= mediana; \%75=3º quartil; Máx. = valores máximos. 
Nas entrevistas a respeito da situação vacinal de cada aluno, um grande número de alunos relatou esquema vacinal incompleto, consideradas as recomendações do PNI; houve melhora da cobertura vacinal conforme a progressão do curso (Tabela 3 ). No caso da varicela, estudo anterior de Yamaguti,
Lima e Santos (2005) revelou que aproximadamente $70 \%$ dos alunos do curso de Medicina relataram ter tido a doença anteriormente, de modo que os valores de cobertura vacinal para esta doença são mais difíceis de serem interpretados.

Tabela 3. Situação de cobertura vacinal relatada por alunos do curso de Medicina da Universidade Estadual de Londrina, matriculados na $1^{\mathrm{a}}, 3^{\mathrm{a}}$ e $6^{\mathrm{a}}$ séries no ano de 2006.

\begin{tabular}{|c|c|c|c|c|}
\hline \multicolumn{5}{|c|}{ Referiram vacinas incompletas } \\
\hline & $\mathbf{1}^{\mathrm{a}}$ série & $3^{a}$ série & $6^{\mathbf{a}}$ série & total \\
\hline $3^{\mathrm{a}}$ dose HepB & $44(58,6 \%)$ & $29(37,1 \%)$ & $6(9,1 \%)$ & $79(36,1 \%)$ \\
\hline SCR & $25(33,3 \%)$ & $19(24,3 \%)$ & $7(10,6 \%)$ & $51(23,3 \%)$ \\
\hline $\mathrm{dT}$ & $16(21,3 \%)$ & $14(17,9 \%)$ & $4(6 \%)$ & $34(15,5 \%)$ \\
\hline
\end{tabular}

\begin{tabular}{|c|c|c|c|c|}
\hline \multicolumn{5}{|c|}{ Referiram vacina contra varicela } \\
\hline & $14(18,7 \%)$ & $26(33,3 \%)$ & $10(15,5 \%)$ & $50(22,8 \%)$ \\
\hline Total de alunos & $75(34,2 \%)$ & $78(35,6 \%)$ & $66(30,1 \%)$ & $219(100 \%)$ \\
\hline
\end{tabular}

Legenda: HepB= contra a Hepatite B; SCR= contra o Sarampo, Caxumba e Rubéola; $\mathrm{dT}=$ dupla tipo adulto, contra difteria e tétano

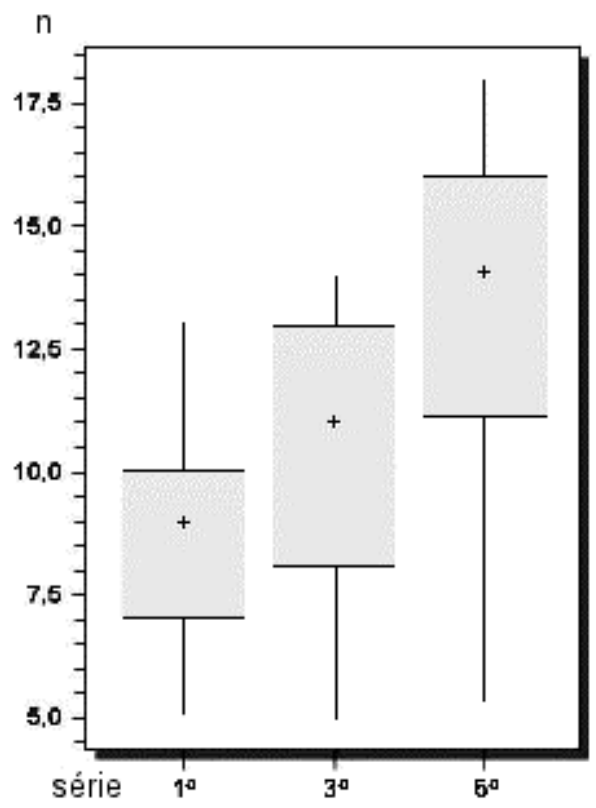

Gráfico 1: Número de respostas corretas dadas por alunos matriculados na $1^{\mathrm{a}}, 3^{\mathrm{a}}$ e $6^{\mathrm{a}}$ séries do curso de Medicina da Universidade Estadual de Londrina no ano de 2006 à pergunta "Para quais destas doenças existem vacinas?", formulada sobre a lista de doenças mostradas no quadro 1. 


\section{Discussão}

Devido ao contato com pacientes ou material infeccioso, muitos profissionais da saúde (médicos, enfermeiros, profissionais da saúde bucal, estudantes, técnicos de laboratório, entre outros) estão constantemente sob o risco de exposição a doenças preveníveis por vacina, assim como podem ser transmissores de tais doenças. (MEDEIROS; MARINO, 2000).

Têm sido relatados na literatura episódios relativos às seguintes doenças: hepatite $\mathrm{B}$, influenza, sarampo, caxumba, rubéola, varicela, hepatite $\mathrm{A}$, difteria, coqueluche, tuberculose e doença meningocócica (ADVISORY COMMITTEE ON IMMUNIZATION PRACTICES, 1997). No presente estudo, as doenças mais citadas pelos alunos quanto ao risco profissional se assemelham aos ditados na literatura: hepatites, HIV/aids, tuberculose, gripe, meningites, pneumonias, sarampo, rubéola e varicela. Os estudantes brasileiros do curso de graduação em Medicina iniciam estágios extracurriculares precoce e desordenadamente, geralmente sem treinamento em biossegurança e sem a verificação de sua situação vacinal. Os riscos de infecção por doenças imunopreveníveis iniciam-se já nos primeiros anos de curso, de modo que não é adequado aguardar-se a entrada no Internato Médico ou na Residência Médica ou no mercado de trabalho para iniciar-se a imunização do médico (PEDROSO et al., 2000; OLIVEIRA; PANIAGO, 2001). Os Ministérios da Educação e Cultura e o da Saúde não possuem uma política específica de imunoprevenção para profissionais de saúde. O Ministério da Saúde apresenta recomendações para algumas situações, sem estarem, no entanto, organizadas em estratégias específicas (BRASIL, 2001). A resolução n ${ }^{\circ} 48 / 2000$ da Agência de Vigilância Sanitária do Ministério da Saúde considera obrigatório que as comissões de Controle de Infecções Hospitalares realizem controle da imunização do pessoal hospitalar, embora as bases técnicas dessa ação não estejam disponíveis para consulta e orientação de conduta (BRASIL, 2001). "A dificuldade de acesso aos comprovantes de vacinação no passado representa uma barreira ao conhecimento real do estado vacinal, e talvez nossos dados fossem diferentes, em relação às vacinas da infância, caso esses comprovantes estivessem disponíveis" (RIBEIRO, 2002). Em nosso estudo, a maioria dos alunos (170 ou 78\%) manifestaram a certeza de possuir carteira de imunização.

A hepatite B provavelmente constitui a doença mais classicamente associada ao exercício de profissões ligadas à área da saúde. O Comitê de Doenças Infecciosas da American Academy of Pediatrics (2000) julga que o momento ideal para vacinação de profissionais de saúde com a vacina contra a hepatite B situa-se antes do início do treinamento prático desses profissionais, recomendação que leva em consideração o maior risco de contaminação do treinando em relação ao profissional mais experiente. Ribeiro (2002) demonstra que os jovens chegam às faculdades desprotegidos, já que a vacina contra hepatite B só passou a ser mais disponível no início da década de 90 do século passado, sendo incorporada ao Calendário Vacinal oficial em 1996, e liberada para uso em adolescentes só em 2002. O presente estudo exemplifica este fato: $44(58,6 \%)$ alunos da $1^{a}$ série referem esquema vacinal incompleto para hepatite $B$.

A vacina tríplice viral (contra Sarampo, Caxumba e Rubéola) só foi incluída no Calendário Básico do PNI a partir de 1996, para menores de 10 anos de idade, aumentando a probabilidade de que os indivíduos nascidos antes de 1986 não estejam vacinados (RIBEIRO, 2002). Em nosso estudo, 51 (23,3\%) dos alunos alegam não ter tomado esta vacina.

Recente trabalho sobre a soroepidemiologia da varicela, realizado em quatro capitais brasileiras, demonstrou que cerca de $8 \%$ dos adultos de 21 a 30 anos de idade são soronegativos (CLEMENTS et al., 1999). Aproximadamente $70 \%$ dos alunos do curso de Medicina da UEL referiram ter tido varicela (YAMAGUTI; LIMA; SANTOS, 2005). A Organização Mundial da Saúde (1999) considera prioritária a vacinação dos susceptíveis maiores de 
15 anos. Entre os alunos da $1^{\mathrm{a}}, 3^{\mathrm{a}}$ e $6^{\mathrm{a}}$ série do curso de Medicina da UEL, 14 (18,7\%), 26 (33,3\%) e 10 $(15,1 \%)$ alunos, respectivamente, alegam certeza de ter recebido vacina contra varicela.

Considera-se que os profissionais de saúde devam ser incentivados a reforçar a imunidade específica para difteria a cada 10 anos (ADVISORY COMMITTEE ON IMMUNIZATION PRACTICES, 1997). Causa preocupação o dado encontrado de que $34(15,5 \%)$ alunos referirem nunca ter recebido a vacina contra difteria e tétano (dT) e apenas $100(45,6 \%)$ referirem ter recebido esta vacina nos últimos 5 anos.

Já foi detectado no Brasil maior risco de infecção pelo bacilo da tuberculose entre profissionais de saúde, inclusive com a participação de bacilos multiresistentes. Recomenda-se que os novos profissionais da saúde sem cicatriz de BCG intradérmica sejam testados com teste intradérmico tuberculínico (PPD) e vacinados caso sejam não reatores (BRASIL, 1994). Apenas 134 (61,2\%) acadêmicos por nós avaliados informam ter a cicatriz da vacina.

No presente estudo, apenas 162 (74\%) dos 219 alunos responderam corretamente quando questionados a respeito de quais recursos procurariam para serem vacinados. As experiências relatadas sugerem que não é suficiente uma simples orientação dos alunos acerca da necessidade da imunização (HASEGAWA; LOPES; GUTIERREZ , 2001; PEDROSO; PEREIRA, 2001). Deve ser implantado um programa mais agressivo de exigência de cumprimento do esquema vacinal, para obtenção de coberturas vacinais adequadas. Medeiros e Marino (2002) relatam que a aceitação das vacinas pelos profissionais é maior quando a vacinação é obrigatória, gratuita e facilmente disponível no local de trabalho; além disso, recomendam exame admissional com história vacinal, sorologia para varicela e teste tuberculínico.

\section{Conclusões}

A maioria dos alunos do curso de Medicina da UEL tem conhecimentos incompletos e, muitas vezes, até incorretos acerca de doenças infecciosas a que estão expostos, risco profissional e doenças imunopreveníveis. Também é restrito o conhecimento acerca das vacinas disponíveis e as que devem receber, inclusive as que já receberam. A cobertura vacinal ainda é falha. Apesar de haver uma evolução desse conhecimento com a progressão do curso, os resultados ainda estão abaixo do ideal. Isto indica a necessidade de delinear e introduzir atividades de esclarecimento sobre risco profissional e de ampliação das coberturas vacinais através de um programa ativo de busca de susceptíveis no curso de Medicina e dos demais cursos da área de saúde da UEL.

\section{Referências}

ADVISORY COMMITTEE ON IMMUNIZATION PRACTICES. General Recommendations on Immunizations. MMWR, Atlanta, v.51, n.2, p.1-36, 2002.

ADVISORY COMMITTEE ON IMMUNIZATION PRACTICES. Immunization of Health Care Workers: Recommendations of the Advisory Committee on Immunization Practices (ACIP) and the Hospital Control Practices Advisory Committee (HICPAC). MMWR, Atlanta, v.469, n.18, p.1-42, 1997.

AMERICAN ACADEMY OF PEDIATRICS. Committee on Infectious Diseases: Hepatitis B. In: 25.ed. Village: Elk Grove, 2000. p.289-302.

BRASIL. Ministério da Saúde. Fundação Nacional da Saúde. Manual dos Centros de Referência de Imunobiológicos Especiais. 2.ed. Brasília, 2001.

BRASIL. Ministério da Saúde. Fundação Nacional de Saúde. Vacinação e/ou revacinação de grupos especiais. In: _____ 2.Informe técnico sobre a vacinação $e$ revacinação BCG. Brasília, 1994. cap.4, p.21-22.

BRASIL. Ministério da Saúde. Resolução Rdc n. 48, 02 de Junho de 2002. Disponível em: <http://elegis . a n vis a.gov.br/le is ref/publi c/ showAct.php?id=6182\&wordझ>. Acesso em: 4 ago. 2006. 
CENTER FOR DISEASECONTROL ANDPREVENTION. Programa computacional de banco de dados e estatística para profissionais de saúde. 2004. Disponível em: <http:/ /www.cdc.gov/EPIINFO/downloads.htm>. Acesso em: 5 abr. 2005.

CLEMENTS, S. A. C.; AZEVEDO, T.; FONSECA, J. C. F., CAVALCANTI, A. M.; SILVEIRA, T. R.; CLEMENS, S. R.. Soroepidemiologia da Varicela no Brasil - resultados de um estudo prospectivo transversal. Jornal de Pediatria, Rio de Janeiro, v.75, n.6, p.433-441, 1999.

HASEGAWA, E. M; LOPES, M H; GUTIERREZ, E B. Avaliação de programa de vacinação para estudantes de Medicina. Boletim da Sociedade Brasileira de Imunizações, São Paulo, v.3, n.4, p.4-8, 2001.

MALUF, E. M. C. P.; ALDENUCCI, M. S. G. Vacinação do Escolar e do Adolescente. In: FARHAT, C. K.; CARVALHO, E. S.; WECKX, L. Y.; CARVALHO, L. H. F. R.; SUCCI, R. C. M. Imunizações: Fundamentos e Prática. 4.ed. São Paulo: Atheneu, 2000. p.205-218.

MEDEIROS, E. A. S.; MARINO, C. G. G. Imunização em profissionais de saúde. In FARHAT, C. K.; CARVALHO, E. S.; WECKX, L. Y.; CARVALHO, L. H. F. R.; SUCCI, R. C. M. Imunizações: Fundamentos e Prática. 4.ed. São Paulo: Atheneu, 2000. p.239-248.

OLIVEIRA, S. M. V. L.; PANIAGO, A. M. M. Exposição ocupacional a sangue e/ou fluídos orgânicos levantamento de 60 casos ocorridos no Hospital Universitário - Universidade Federal do Mato Grosso do Sul. In: CONGRESSODA SOCIEDADE BRASILEIRADE MEDICINA TROPICAL, 2001, Salvador. Revista da Sociedade Brasileira de Medicina Tropical, Rio de Janeiro, v.34, p.418, 2001, supl.1.
PEDROSO, E. R. P; LAMOUNIER FILHO, A.; RAMPINELLI, C. A.; REIS, J. M. B.. Estudo descritivo dos acidentes com material potencialmente contaminado por sangue em ambiente hospitalar entre estudantes de medicina. In: CONGRESSO DA SOCIEDADE BRASILEIRA DE MEDICINA TROPICAL, 36., 2000, São Luiz. Revista da Sociedade Brasileira de Medicina Tropical, Rio de Janeiro, v.33, p.257-258, 2000. Supl.1.

PEDROSO, E. R. P; PEREIRA, E. M. Prevalência da vacinação entre acadêmicos de Medicina da Universidade Federal de Minas Gerais. In: CONGRESSO DA SOCIEDADE BRASILEIRA DA MEDICINA TROPICAL, 37., 2001, Salvador. Revista da Sociedade Brasileira de Medicina Tropical, Rio de Janeiro, v.34, p.444-445, 2001. Supl. 1.

RIBEIRO, J. G. L. Necessidade de adição de uma política específica de imunização para acadêmicos de medicina: a situação da Faculdade de Ciências Médicas de Minas Gerais. 2002. Dissertação (Mestrado) - Faculdade de Medicina da Universidade Federal de Minas Gerais, Belo Horizonte.

WORLD HEALTH ORGANIZATION. Varicella vaccine. Weekly Epidemiological Record. Genebra, v.73, n.32, p.1$5,1999$.

YAMAGUTI, H. Y.; LIMA, G. Z.; SANTOS, A. C. J. Conhecimentos sobre varicela de alunos em cursos da área de saúde na Universidade Estadual de Londrina e verificação da presença de cicatrizes da doença. In ENCONTRO ANUAL DE INICIAÇÃO CIENTÍFICA, 15. 2005, Guarapuava. Apresentação Oral... Guarapuava: Universidade Estadual do Centro Oeste, 2005. 